ANNALES

POLONICI MATHEMATICI

$97.2(2010)$

\title{
Algebraic approximation of analytic sets definable in an o-minimal structure
}

\author{
by Marcin Bilski and Kamil Rusek (Kraków)
}

\begin{abstract}
Let $K, R$ be an algebraically closed field (of characteristic zero) and a real closed field respectively with $K=R(\sqrt{-1})$. We show that every $K$-analytic set definable in an o-minimal expansion of $R$ can be locally approximated by a sequence of $K$-Nash sets.
\end{abstract}

1. Introduction. Let $K$ be an algebraically closed field (of characteristic zero) and let $R$ be a real closed field such that $K=R(\sqrt{-1})$. In [11], [12] Y. Peterzil and S. Starchenko developed the idea of $K$-differentiable functions definable in an o-minimal expansion of $R$, which allowed them to extend the notion of classical $\mathbb{C}$-analyticity to a non-standard context. It turned out that many interesting properties of $\mathbb{C}$-analytic functions remain true in this new setting and some of these appear in stronger versions. The methods used in [11] and [12] differ from the classical treatment of analytic functions and are similar in spirit to the topological approach of Whyburn (see [19]). This is because neither converging power series nor integrals can be used in the non-standard context.

The notion of a $K$-analytic set (i.e. a set locally described by a finite number of $K$-differentiable functions) was first introduced in [12] and the theory of such sets was developed in [13. It became clear that, in the presence of an o-minimal structure, the basic theorems of complex analytic geometry have their non-standard counterparts which are often essentially stronger. For example, every definable $K$-analytic subset of $K^{n}$ is $K$-algebraic (extending the classical Chow theorem) and the image of a definable $K$-analytic set under a projection onto a definable $K$-manifold must be $K$-analytic if it is closed (see [13]). The latter fact generalizes the Remmert proper mapping theorem. In the standard setting, the existence of proper projections of analytic sets along affine spaces is a local property (generally holding in some neigh-

2010 Mathematics Subject Classification: 03C64, 14P99.

Key words and phrases: $K$-Nash set, $K$-analytic set, o-minimal structure. 
borhood of a given point). Definability helps to globalize it in some sense (see [13, Theorem 2.13]). Similarly to the classical situation, every definable pure-dimensional $K$-analytic set can be locally represented as an analytic branched covering.

The aim of the present paper is to show that $K$-analytic sets definable in an o-minimal expansion of $R$ admit local approximation by $K$-Nash sets, which extends some of recent results obtained for classical analytic sets (see [2], 3], 44). Since in the non-standard case the Taylor series do not generally converge we cannot hope to obtain the local uniform algebraic approximation of $K$-differentiable functions by analogy to the classical situation. However, the sum of the first terms of the Taylor expansion approximates a given function as a tangent polynomial with the prescribed order of tangency (see Theorem 2.2). In this paper we show that for any $K$-analytic set definable in an o-minimal expansion of $R$ there exist higher order tangent $K$-Nash sets. The o-minimal structure will be fixed throughout the article and by "definable" we mean definable (possibly with parameters) in this structure.

Denote $\left\|\left(z_{1}, \ldots, z_{m}\right)\right\|_{K^{m}}=\max _{i=1, \ldots, m}\left|z_{i}\right|$ (in what follows, the subscript $K^{m}$ will often be omitted). For definable closed bounded subsets $A, B$ of $K^{m}$ we denote by $\operatorname{dist}(A, B)$ the Hausdorff distance between $A, B$.

Let $X$ be a definable $K$-analytic subset of a definable open $U \subset K^{m}$. Assume that $X$ is of pure $K$-dimension $n$ and that $\mathbf{0} \in X$. There exists a $K$-linear subspace $L$ of $K^{m}$ of dimension $m-n$ such that the intersection of $L$ and $X$ at $\mathbf{0}$ is isolated (follows immediately from Theorem 2.13 and Corollary 5.2 of [13]). Take any such space and denote by $L^{\perp}$ its orthogonal complement. Then the projection of $X$ onto $L^{\perp}$ is a $K$-analytic branched covering in some neighborhood of $\mathbf{0}$ (see Theorem 2.6 below). Let $d$ be the multiplicity of this projection at $\mathbf{0}$ (for a definition see Section 2.2).

Assuming the notation of Section 2 we prove

TheOREM 1.1. There are open balls $B^{\prime} \subset L^{\perp}, B^{\prime \prime} \subset L$ and a sequence $\left\{X_{\nu}\right\}$ of $K$-Nash subsets of $B^{\prime} \times B^{\prime \prime}$ of pure $K$-dimension $n$ such that $\mathbf{0} \in$ $B^{\prime} \times B^{\prime \prime}$ and for every $\nu \in \mathbb{N}$ the projections of $X_{\nu}$ and $X \cap\left(B^{\prime} \times B^{\prime \prime}\right)$ onto $B^{\prime}$ are $d$-sheeted $K$-analytic branched coverings. Moreover,

$$
\operatorname{dist}\left(X \cap\left(\{x\} \times B^{\prime \prime}\right), X_{\nu} \cap\left(\{x\} \times B^{\prime \prime}\right)\right) \leq\|x\|^{\nu}
$$

for every $x \in B^{\prime}$ and $\nu \in \mathbb{N}$.

In fact, we prove a slightly stronger Theorem 4.1 which immediately implies the result above. As in standard analytic geometry, the basic obstacle in the proof comes from the fact that there exist analytic sets for which the number of describing functions is greater than the codimension. Obviously one cannot approximate such sets by approximating the describing functions arbitrarily because generic approximation of these functions yields sets of 
dimension smaller than required. For classical analytic sets this difficulty can be overcome by using the Lempert theorem on approximation of analytic solutions of Nash equations (see [8]). Here we shall formulate and prove a local analogue of this theorem in the non-standard setting (see Theorem 3.1).

The organization of this paper is as follows. In Section 2 we give preliminary material about $K$-Nash sets, $K$-analytic sets and symmetric powers. In the next section we prove facts concerning approximation of $K$-analytic mappings between singular spaces, which will be used in Section 4 where the proofs of our main results are completed.

\section{Preliminaries}

2.1. $K$-differentiable functions, $K$-Nash functions. $K$-differentiable functions were first introduced in [11] (the case of one variable) and in [12] (several variables). Let us recall the definition. Let $V \subset K^{n}$ be a definable open set. Then a definable map $F: V \rightarrow K$ is called $K$-differentiable on $V$ if it is continuous on $V$ and for every $\left(z_{1}, \ldots, z_{n}\right) \in V$ and $i=1, \ldots, n$ the function $F\left(z_{1}, \ldots, z_{i-1}, \cdot, z_{i+1}, \ldots, z_{n}\right)$ is $K$-differentiable in the $i$ th variable at $z_{i}$.

The following facts from [12] will be useful:

Theorem 2.1. Let $L \subset V$ be definable sets, where $V$ is an open subset of $K^{n}$ and the o-minimal dimension of $L$ is strictly smaller than $2 n-1$. Assume that $F: V \backslash L \rightarrow K$ is a definable $K$-differentiable function that is bounded in a neighborhood of every $u \in L$. Then $F$ can be extended uniquely to a definable $K$-differentiable function on $V$.

Proof. See [12, Theorem 2.15].

Let us also recall that the Weierstrass preparation and division theorems hold for the ring of germs of definable $K$-differentiable functions. (For proofs see [12, Theorems 2.20 and 2.23].)

Finally, let $T_{a}^{k} f$ denote the sum of the terms of the Taylor expansion of the function $f$ in a neighborhood of $a$ of order not exceeding $k$.

TheOREM 2.2. Let $D$ be an open definable subset of $K^{n}$ containing the closed unit ball $B$ centered at $0 \in K^{n}$ and let $f: D \rightarrow K$ be a definable $K$-differentiable function. Assume also that $|f(z)| \leq 1$ for all $z \in B$. Then

$$
\left|f(z)-T_{0}^{k-1} f(z)\right| \leq\|2 z\|^{k}
$$

for all $k \geq 1$ and $z \in B$.

Proof. For the case $n=1$ see [11, Theorem 2.50]. Assume $n>1$, fix $k \in \mathbb{N}$ and take any $z \in K^{n}$ with $\|z\|<1$. Then there are $t_{0} \in K$ with $\left|t_{0}\right|<1$ and $\tilde{z} \in K^{n}$ such that $\|\tilde{z}\|=1$ and $z=\tilde{z} \cdot t_{0}$. Now apply the 
theorem to the function $\tilde{f}(t)=f(\tilde{z} \cdot t)$ for $t$ from a closed unit disc in $K$. We obtain

$$
\left|\tilde{f}(t)-T_{0}^{k-1} \tilde{f}(t)\right| \leq|2 t|^{k} .
$$

Put $t=t_{0}$. As $\left|t_{0}\right|=\|z\|, \tilde{f}\left(t_{0}\right)=f(z)$ and $T_{0}^{k-1} \tilde{f}\left(t_{0}\right)=T_{0}^{k-1} f(z)$ we get our claim.

Let $\Omega$ be a definable open subset of $K^{n}$. Assume that $\Omega$ is definably connected. Let $f$ be a definable $K$-differentiable function on $\Omega$. We say that $f$ is a $K$-Nash function if there exists a polynomial $P: K^{n} \times K \rightarrow K, P \neq 0$, such that $P(x, f(x))=0$ for $x \in \Omega$. A definable continuous mapping on $\Omega$ with values in $K^{N}$ is said to be a $K$-Nash mapping if each of its components is a $K$-Nash function.

THEOREM 2.3. The set of all $K$-Nash functions on $\Omega$ is a ring.

Proof. Identical to the proof in the classical complex case (see [17]).

Finally, let us observe that the Weierstrass preparation and division theorems remain true in the class of $K$-Nash functions. As for the preparation theorem, in view of the existence of such a theorem for definable $K$ differentiable functions, it suffices to check the following claim. Let $f\left(z^{\prime}, z_{n}\right)$ be a non-zero $K$-Nash function defined in some definably connected neighborhood $U \times V$ of zero in $K^{n-1} \times K$ such that $f=H W$, where $H$ is a definable $K$-differentiable non-vanishing function and $W\left(z^{\prime}, z_{n}\right)=z_{n}^{s}+z_{n}^{s-1} a_{1}\left(z^{\prime}\right)+$ $\cdots+z_{n} a_{s-1}\left(z^{\prime}\right)+a_{s}\left(z^{\prime}\right)$, where $a_{1}, \ldots, a_{s}$ are definable $K$-differentiable functions and $W^{-1}(0) \subset U \times V$. Then $a_{1}, \ldots, a_{s}, H$ are $K$-Nash functions.

To prove the claim, put $z=\left(z^{\prime}, z_{n}\right)$ and take a non-zero polynomial $P(z, u)$ such that $P(z, f(z))=0$ in $U \times V$. Since $f$ is a non-zero function, we may assume that $P(z, u)=u^{r} b_{0}(z)+\cdots+u b_{r-1}(z)+b_{r}(z)$, where $b_{0}, \ldots, b_{r}$ are polynomials and $b_{r} \neq 0$ (because if $b_{r}=0$ we may replace $P$ by $P / u$ ).

Now $\{f=0\} \subset\left\{b_{r}=0\right\}$. On the other hand, there is an open ball $B \subset U$ such that $\left.W\right|_{B \times K}=\left(z_{n}-A_{1}\left(z^{\prime}\right)\right) \cdots\left(z_{n}-A_{s}\left(z^{\prime}\right)\right)$, where $A_{1}, \ldots, A_{s}$ are definable $K$-differentiable functions. Since $\operatorname{graph}\left(A_{j}\right) \subset\left\{b_{r}=0\right\}$, the function $A_{j}$ is $K$-Nash for $j=1, \ldots, s$. This implies that $\left.a_{1}\right|_{B}, \ldots,\left.a_{s}\right|_{B}$ are $K$-Nash functions and hence so are $a_{1}, \ldots, a_{s}$ by the identity principle, in view of the fact that $U$ is definably connected.

Let us turn to the division theorem. Let $f\left(z^{\prime}, z_{n}\right)$ be a non-zero $K$-Nash function defined in some definably connected neighborhood $U \times V$ of zero in $K^{n-1} \times K$, and let $W\left(z^{\prime}, z_{n}\right)=z_{n}^{s}+z_{n}^{s-1} a_{1}\left(z^{\prime}\right)+\cdots+z_{n} a_{s-1}\left(z^{\prime}\right)+a_{s}\left(z^{\prime}\right)$, where $a_{1}, \ldots, a_{s}$ are $K$-Nash functions and $W^{-1}(0) \subset U \times V$. We need to show that if $H$ and $r$ are a definable $K$-differentiable function and a polynomial with definable $K$-differentiable coefficients, respectively, such that $f=W H+r$ and $\operatorname{deg}(r)<s$, then both $H$ and $r$ are $K$-Nash functions. In fact, it is sufficient to check that the coefficients of $r$ are $K$-Nash functions. We argue 
by induction on $\operatorname{deg}(W)$. If $s=1$ then $W\left(z^{\prime}, z_{n}\right)=z_{n}-A\left(z^{\prime}\right)$, where $A$ is a $K$-Nash function and $r$ depends only on $z^{\prime}$, therefore $r\left(z^{\prime}\right)=f\left(z^{\prime}, A\left(z^{\prime}\right)\right)$ is a $K$-Nash function.

Suppose $s>1$. Then there is an open ball $B \subset U$ such that $\left.W\left(z^{\prime}, z_{n}\right)\right|_{B \times K}$ $=\left(z_{n}-A\left(z^{\prime}\right)\right) \tilde{W}\left(z^{\prime}, z_{n}\right)$ and $\tilde{W}$ is a polynomial with $K$-Nash coefficients and $A\left(z^{\prime}\right)$ is a $K$-Nash function. Applying the division theorem for $K$ differentiable functions we obtain $f=\tilde{W} \tilde{H}+\tilde{r}$ and $\tilde{H}=\left(z_{n}-A\left(z^{\prime}\right)\right) \bar{H}+\bar{r}$ on $B \times K$ where $\operatorname{deg}(\tilde{r})<s-1$ and $\operatorname{deg}(\bar{r})=0$. By the induction hypothesis both $\tilde{r}$ and $\bar{r}$ are $K$-Nash functions on $B \times K$. Moreover, $\left.r\right|_{B \times K}=\tilde{W} \bar{r}+\tilde{r}$, so the coefficients of $\tilde{r}$ are $K$-Nash functions on $B$, hence (as above) on $U$.

2.2. $K$-analytic sets, $K$-Nash sets. We recall the basic notions of $K$-analytic geometry. Its main objects, $K$-analytic sets, were introduced in [12] as subsets of definable manifolds. Here we restrict our attention to the case of subsets of $K^{n}$.

Let $Y \subset V$ be definable subsets of $K^{n}$, with $V$ open in $K^{n}$, and $Y$ closed in $V$. We say that $Y$ is a $K$-analytic [K-Nash] subset of $V$ if for every $y_{0} \in Y$ there is a definable open neighborhood $U$ of $y_{0}$ and a $K$-differentiable [K-Nash] mapping $f: U \rightarrow K^{m}$ for some $m \in \mathbb{N}$ such that $Y \cap U=f^{-1}(0)$. Then $\operatorname{Reg}_{K}(Y)$ denotes the set of all points in $Y$ in some neighborhood of which $Y$ is a $K$-differentiable manifold.

In the following, for any definable $K$-analytic set $Y$ we denote by $\operatorname{dim}_{K}(Y)$ the $K$-dimension of $Y$, whereas for any definable set $Y, \operatorname{dim}_{R}(Y)$ denotes the o-minimal dimension of $Y$. Clearly, $\operatorname{dim}_{R}(Y)=2 \operatorname{dim}_{K}(Y)$ for any definable $K$-analytic set.

The following definition will be useful. Let $X \subset R^{m}$ and $Y \subset R^{n}$ be definable sets and let $f: X \rightarrow Y$ be a definable continuous mapping. We say that $f$ is definably proper if for every $y \in Y$ and every definable curve $\gamma:(0,1) \rightarrow X$ such that $f(\gamma(t)) \rightarrow y$ as $t \rightarrow 0, \gamma(t)$ has a limit in $X$ for $t \rightarrow 0$. This could be equivalently restated as: for each definable set $K \subset Y$, if $K$ is closed and bounded in $R^{n}$ then $f^{-1}(K)$ is closed and bounded in $R^{m}$ (see [7, p. 104]).

The following theorem contains a definable analogue of the classical Remmert theorem which is an immediate consequence of its more general version (see [13, Theorem 7.4]). Let $\pi: V \times K^{k} \rightarrow V$ denote the natural projection, where $V$ is open and definable in an o-minimal expansion of $R$.

TheOrem 2.4. Let $X$ be a definable $K$-analytic $[K-N a s h]$ subset of $V \times K^{k}$ such that $\left.\pi\right|_{X}: X \rightarrow V$ is a definably proper mapping. Then $\pi(X)$ is a definable $K$-analytic $[K-N a s h]$ subset of $V$ and $\operatorname{dim}_{K}(X)=\operatorname{dim}_{K}(\pi(X))$.

Proof. Definable properness ensures uniform finiteness of the projection $\left.\pi\right|_{X}$. Indeed, for every $y \in V$ the set $\pi^{-1}(\{y\})$ is finite (because it is 
definable bounded and $K$-analytic, see [13]). Then definability implies uniform finiteness (see [7, p. 60]). Thus by the definition of o-minimal dimension we immediately have $\operatorname{dim}_{R}(X)=\operatorname{dim}_{R}(\pi(X))$, so $\operatorname{dim}_{K}(X)=\operatorname{dim}_{K}(\pi(X))$.

One easily checks that $\pi(X)$ is a definable closed subset of $V$, hence by Theorem 7.4 from [13], $\pi(X)$ is a definable $K$-analytic subset of $V$.

Recall that the classical proof of the Remmert theorem for complex analytic sets involves only the Weierstrass preparation and division theorems and the computation of the resultants of polynomials obtained by applying these theorems. Therefore to repeat this proof in the case where $X$ is a $K$ Nash set it is sufficient to know that the Weierstrass theorems remain true in the class of $K$-Nash functions, which has been checked in the previous subsection. (Clearly, the Remmert theorem for $K$-analytic sets can also be obtained in this way.)

The following fact explains the relation between Nash and algebraic sets.

TheOREM 2.5. Let $X$ be an irreducible $K$-Nash subset of a definable open $\Omega \subset K^{n}$. Then there exists an algebraic subset $Y$ of $K^{n}$ such that $X$ is a $K$-analytic irreducible component of $Y \cap \Omega$. Conversely, every $K$-analytic irreducible component of $Y \cap \Omega$ is an irreducible $K$-Nash subset of $\Omega$.

Proof. Identical to the proof in the classical complex case (see [17, Section 2.B]).

Purely $m$-dimensional $K$-analytic sets with definably proper projection onto a subdomain of $K^{m}$ are branched coverings:

THEOREM 2.6. Let $U$ be an open definably connected subset of $K^{m}$ and let $X$ be a definable $K$-analytic subset of $U \times K^{k}$ of pure $K$-dimension $m$ such that the projection $\left.\pi\right|_{X}: X \rightarrow U$ is definably proper. Then the mapping $\left.\pi\right|_{X}: X \rightarrow U$ is surjective and open. There exist an integer $s=s(X)$ and $a$ definable subset $S=S(X)$ of $U$ with $\operatorname{dim}_{R}(S) \leq 2 m-2$ such that

$$
\sharp\left(\left.\pi\right|_{X}\right)^{-1}(x) \begin{cases}=s & \text { for } x \in U \backslash S, \\ <s & \text { for } x \in S .\end{cases}
$$

Moreover, for every $x \in U \backslash S$ there is a neighborhood $V \subset U \backslash S$ of $x$ and definable $K$-analytic mappings $f_{1}, \ldots, f_{s}: V \rightarrow K^{k}$ such that $f_{i} \cap f_{j}=\emptyset$ for $i \neq j$, and $X \cap\left(V \times K^{k}\right)=f_{1} \cup \cdots \cup f_{s}$.

Proof. Immediate consequence of Lemmas 3.5, 4.5 and 4.7 of [13].

TheOrem 2.7. Let $A$ be a purely $n$-dimensional $K$-analytic subset of $K^{m}$, and let $a \in A, n<m$. Then there is an $(m-n)$-dimensional $K$-affine subspace $L$ of $K^{m}$ such that $a$ is an isolated point of $L \cap A$. For every affine subspace $L$ of $K^{m}$ such that $\operatorname{dim}_{K}(L)>m-n$ and $a \in L$ we have $\operatorname{dim}_{K}(L \cap X)>0$. 
Proof. Since $n<m$, there is a one-dimensional $K$-space $L^{\prime}$ passing through $a$ not contained in $X$, hence intersecting $X$ in isolated points. If $m-n=1$ the proof of the first claim is complete. Otherwise, pick $e_{1} \in L^{\prime}$, $e_{1} \neq 0$. Then there is a neighborhood $V$ of $a$ in $K^{m}$ in which the projection $\pi_{L^{\prime}}$ of $X$ along $L^{\prime}$ onto $L^{\prime \perp}$ is definably proper, where $L^{\prime \perp}$ denotes the orthogonal complement of $L^{\prime}$ in $K^{m}$. By Theorem 2.4. $\pi_{L^{\prime}}(V \cap X)$ is a definable $K$-analytic subset of some open set in $L^{\prime \perp}$ of pure dimension $n$. By an inductive argument (on $m$ ) we may assume that there is an $(m-n-1)$-dimensional $K$-affine subspace $M \subset L^{\prime \perp}$ intersecting $\pi_{L^{\prime}}(X)$ at the isolated point $\pi_{L^{\prime}}(a)$. The space spanned by $M$ and $e_{1}$ satisfies the first claim of the theorem.

If there were a $K$-affine space $L$ of $K^{m}$ of dimension larger than $m-n$ with an isolated intersection of $X$ at $a$, the projection of $X$ along $L$ onto $L^{\perp}$ would be definably proper in some neighborhood of $a$. Then the fact that $X$ is $n$-dimensional contradicts Theorem 2.4, as $L^{\prime \perp}$ is at most $(n-1)$ dimensional.

Let $A$ be a purely $n$-dimensional $K$-analytic subset of $K^{m}$ and let $L$ be an $(m-n)$-dimensional $K$-affine subspace of $K^{m}$ such that $a$ is an isolated point of $L \cap A$. Then there is a domain $U \subset K^{m}$ such that $U \cap A \cap L=\{a\}$ and the projection $\pi_{L}: U \cap A \rightarrow \pi_{L}(U) \subset L^{\perp}$ along $L$ is a $k$-sheeted $K$ analytic branched covering (see Theorem 2.6). The number $k$ will be called the multiplicity of $\pi_{L}$ at $a$ and denoted by $\mu_{a}\left(\left.\pi_{L}\right|_{A}\right)$ (cf. [5, pp. 102, 120], for the classical counterpart).

2.3. Symmetric powers. Let $\left(K^{k}\right)_{\mathrm{sym}}^{d}$ and $\left\langle x_{1}, \ldots, x_{d}\right\rangle$ denote $\left(K^{k}\right)^{d /} \sim$ and the equivalence class of $\left(x_{1}, \ldots, x_{d}\right) \in\left(K^{k}\right)^{d}$ respectively, where $\left(x_{1}^{\prime}, \ldots, x_{d}^{\prime}\right) \sim\left(x_{1}, \ldots, x_{d}\right)$ if and only if $\left(x_{1}^{\prime}, \ldots, x_{d}^{\prime}\right)=\left(x_{p(1)}, \ldots, x_{p(d)}\right)$ for some permutation $p$. We endow $\left(K^{k}\right)_{\text {sym }}^{d}$ with a metric $\rho_{k}$ given by

$$
\rho_{k}\left(\left\langle x_{1}, \ldots, x_{d}\right\rangle,\left\langle y_{1}, \ldots y_{d}\right\rangle\right)=\inf _{p} \sup _{i}\left\|x_{i}-y_{p(i)}\right\|_{K^{k}},
$$

where $\left\|\left(z_{1}, \ldots, z_{k}\right)\right\|_{K^{k}}=\max _{i=1, \ldots, k}\left|z_{i}\right|$, whereas $p$ is any permutation of $(1, \ldots, d)$ (the subscript $k$ in $\rho_{k}$ will often be omitted).

Then there exist an integer $N$ and a mapping $\phi:\left(K^{k}\right)_{\text {sym }}^{d} \rightarrow K^{N}$ with the following properties:

(a) $\phi$ is injective and $\phi, \phi^{-1}$ are continuous,

(b) $\phi \circ \pi_{\text {sym }}:\left(K^{k}\right)^{d} \rightarrow K^{N}$ is a definably proper polynomial mapping, where $\pi_{\mathrm{sym}}\left(x_{1}, \ldots, x_{d}\right)=\left\langle x_{1}, \ldots, x_{d}\right\rangle$,

(c) $\phi\left(\left(K^{k}\right)_{\mathrm{sym}}^{d}\right)$ is an algebraic subset of $K^{N}$.

The proofs of (a) and (b) are the same as in the classical case (cf. [18, pp. 366-368, 152-154]). As for (c), in view of (b), by the Remmert theorem (see Theorem 2.4 $\phi\left(\left(K^{k}\right)_{\mathrm{sym}}^{d}\right)$ is a definable global $K$-analytic subset of $K^{N}$, hence algebraic (see [13, Theorem 5.1]). 
3. Algebraic approximation of $K$-differentiable mappings. The aim of this section is to obtain the definable analogue of the Lempert theorem on approximation of holomorphic solutions of Nash equations by Nash solutions (see [8, Theorem 3.2]). In the real case such a result is due to M. Coste, J. Ruiz and M. Shiota ([6, Theorem 1.1]).

Let $B_{n}(r)=\left\{x \in K^{n}:\|x\|<r\right\}$. We prove

Theorem 3.1. Let $f: B_{n}(r) \rightarrow K^{m}$ be a definable $K$-differentiable mapping which satisfies a system of equations

$$
Q(z, f(z))=0 \quad \text { for every } z \in B_{n}(r) .
$$

Here $Q$ is a $K$-Nash mapping from a neighborhood of the graph of $f$ into some $K^{q}$. Then there are $0<s \leq r$ and a sequence $f_{\nu}: B_{n}(s) \rightarrow K^{m}$ of $K$-Nash mappings such that

$$
Q\left(z, f_{\nu}(z)\right)=0 \quad \text { and } \quad\left\|f_{\nu}(z)-f(z)\right\| \leq\|z\|^{\nu}
$$

for every $z \in B_{n}(s)$ and $\nu \in \mathbb{N}$.

Proof of Theorem 3.1. Similarly to [6] and [8] the main ingredient of the proof is an application of the following

TheOREM 3.2. Let $W$ be an algebraic subset of $K^{m}$ and let $h: B_{n}(r) \rightarrow W$ be a $K$-differentiable mapping. Then there are $0<s \leq r$, a non-singular algebraic subset $V$ of $K^{p}$, a $K$-differentiable mapping $f: B_{n}(s) \rightarrow V$ and a polynomial mapping $g: V \rightarrow W$ such that $\left.h\right|_{B_{n}(s)}=g \circ f$.

Proof of Theorem 3.2. Let $\mathcal{O}_{n}$ denote the ring of germs of $K$-differentiable functions (defined in some neighborhood of $0 \in K^{n}$ ) at $0 \in K^{n}$. Functions and their germs will be denoted by the same letters.

First we recall the basic properties of the $\operatorname{ring} \mathcal{O}_{n}$ from [12]:

Proposition 3.3.

(1) $\mathcal{O}_{n}$ is an integral domain,

(2) $u$ is a unit of $\mathcal{O}_{n}$ if and only if $u(0) \neq 0$,

(3) $\mathcal{O}_{n}$ has a unique maximal ideal $I_{n}$, which is the set of all germs $f$ such that $f(0)=0$,

(4) the map which associates to every $f \in \mathcal{O}_{n}$ the formal power series of its partial derivatives at 0 is an embedding of $\mathcal{O}_{n}$ into the ring of all formal power series in the variables $x_{1}, \ldots, x_{n}$ over $K$,

(5) $\mathcal{O}_{n}$ is Noetherian.

Now observe that $\left(\mathcal{O}_{n}, I_{n}\right)$ is a regular local ring. Indeed, by Proposition $3.3(4)$, and since $\mathcal{O}_{n}$ contains the germs of polynomials in $x_{1}, \ldots, x_{n}$ over $K$, it follows that the $I_{n}$-adic completion $\widehat{\mathcal{O}}_{n}$ equals $K\left[\left[x_{1}, \ldots, x_{n}\right]\right]$. Hence $\widehat{\mathcal{O}}_{n}$ is regular and so is $\mathcal{O}_{n}$. Consequently, the inclusion $e: K \hookrightarrow \mathcal{O}_{n}$ is a regular homomorphism (for the definition see [9, p. 249]). 
Here we invoke a theorem of D. Popescu ([14], [15]; see also [16], [1], [10]):

TheOrem 3.4. Let $\sigma: A \rightarrow B$ be a homomorphism of Noetherian rings. Then the following conditions are equivalent:

(1) $\sigma$ is regular,

(2) for every A-algebra $C$ of finite type and for every A-homomorphism $s: C \rightarrow B$ there are an $A$-homomorphism $\lambda: C \rightarrow D$, where $D$ is a smooth A-algebra of finite type, and an A-homomorphism $\chi: D \rightarrow B$ such that $\chi \circ \lambda=s$. (Here the ring $B$ is treated as an A-algebra via the homomorphism $\sigma$.)

We apply this theorem in the following setting. Take $\sigma$ to be our regular homomorphism $e: K \hookrightarrow \mathcal{O}_{n}$ and define $s: K[W] \rightarrow \mathcal{O}_{n}$ by $s(u)=u \circ h$. Then there is a non-singular algebraic subset $V$ of $K^{p}$ and $K$-homomorphisms $\Phi: K[W] \rightarrow K[V], \Psi: K[V] \rightarrow \mathcal{O}_{n}$ such that $s=\Psi \circ \Phi$. Clearly, $\Phi$ and $\Psi$ are induced by a polynomial mapping $g: V \rightarrow W$ and a $K$-differentiable mapping $f: B_{n}(s) \rightarrow V$ respectively so the germ of $g \circ f$ equals the germ of $h$.

Proof of Theorem 3.1 (continuation). Denote by $W$ a $K$-algebraic subset of $K^{n+m}$ such that in a neighborhood of $(0, f(0))$ the set $\left\{(x, y) \in K^{n+m}\right.$ : $Q(x, y)=0\}$ is the union of some $K$-analytic irreducible components of $W$. (The existence of $W$ follows from Theorem 2.5.) Consider the mapping

$$
F: B_{n}(r) \ni z \mapsto(z, f(z)) \in W \subset K^{n+m} .
$$

Next apply Theorem 3.2 to obtain a $K$-algebraic subset $Z$ of $K^{p}$, a polynomial mapping $G: Z \rightarrow W$ and a definable $K$-differentiable mapping $H: B_{n}(s) \rightarrow Z$ such that $\operatorname{im} H \subset \operatorname{Reg}_{K}(Z)$ and $\left.F\right|_{B_{n}(s)}=G \circ H$.

Observe that without loss of generality we may assume that $Z=K^{p}$, $H\left(B_{n}(s)\right) \subset B_{p}(\bar{s}), \bar{s}>0$ and $G: B_{p}(\bar{s}) \rightarrow W$ is a Nash mapping (this is a simple consequence of the fact that $\operatorname{Reg}_{K}(Z)$ is locally $K$-Nash equivalent to a germ of an affine space).

Now by Theorem 2.2 we have a sequence $H_{\nu}: B_{n}(s) \rightarrow K^{p}$ of $K$-polynomial mappings such that $\left\|H_{\nu}(z)-H(z)\right\| \leq\|z\|^{\nu}$ for every $z \in B_{n}(s)$, $\nu \in \mathbb{N}$ (decreasing $s$ if necessary). It is easy to see that $K$-differentiability and definability of $G$ give

$$
\left\|(G \circ H)(z)-\left(G \circ H_{\nu}\right)(z)\right\| \leq\|z\|^{\nu}
$$

(after renumbering the sequence $H_{\nu}$ and again decreasing $s$ if necessary).

Since the first $n$ components of $G \circ H$ is the identity mapping, we may assume, by the Inverse Function Theorem, that the first $n$ components of $G \circ H_{\nu}$ is also the identity mapping (otherwise we compose it with its inverse). Finally, one observes that if $\nu$ is large enough then

$$
\operatorname{im}\left(G \circ H_{\nu}\right) \subset\left\{(x, y) \in K^{n+m}: Q(x, y)=0\right\} .
$$


This follows from the fact that $K$-analytic irreducible components of $W \cap \Omega$, where $\Omega \subset K^{n+m}$ is an open ball, are semialgebraic, hence their order of tangency is bounded.

Now it is sufficient to take $f_{\nu}$ to be the last $m$ components of $G \circ H_{\nu}$.

4. Approximation of $K$-analytic sets by $K$-Nash sets. In what follows, "dimension" always means $K$-dimension. Let $A, B$ be finite subsets of $K^{k}$ and let $d$ be a fixed positive integer. Suppose that for all $a \in A$ and $b \in B$ there are positive integers $n_{a, A}, n_{b, B}$ such that $\sum_{a \in A} n_{a, A}=\sum_{b \in B} n_{b, B}=d$. Then the set $A$ can be treated as a tuple from $\left(K^{k}\right)_{\text {sym }}^{d}$ (see Section 2.3 for definition), where every $a$ appears in the tuple $n_{a, A}$ times (the same holds true for $B)$. We denote by $\rho(A, B)$ the distance between the sets $A, B \in\left(K^{k}\right)_{\text {sym }}^{d}$ as defined in Section 2.3. In Theorem 4.1 below, $A, B$ will be the fibers of proper projections of $K$-analytic subsets of $B_{m}(t) \times K^{k}$ of pure $K$-dimension $m$ onto $B_{m}(t)$, where $B_{m}(t)=\left\{z \in K^{m}:\|z\|_{K^{m}}<t\right\}$. Then the numbers $n_{a, A}, n_{b, B}$ will be, by definition, the multiplicities of these projections at $a, b$ respectively (for the notion of the multiplicity of a projection see Section 2.2.

Assuming the notation of Section 2 we prove

THeOREm 4.1. Let $X$ be a definable $K$-analytic subset of $B_{m}(1) \times K^{k}$ of pure $K$-dimension $m$ with definably proper projection onto $B_{m}(1)$. Let $d$ denote the cardinality of the generic fiber of $X$ over $B_{m}(1)$. Then there are $0<t \leq 1$ and a sequence $\left\{X_{\nu}\right\}$ of $K$-Nash subsets of $B_{m}(t) \times K^{k}$ of pure $K$-dimension $m$ such that for every $\nu \in \mathbb{N}$ the generic fiber of $X_{\nu}$ over $B_{m}(t)$ has d elements. Moreover,

$$
\rho\left(\left(\{x\} \times K^{k}\right) \cap X,\left(\{x\} \times K^{k}\right) \cap X_{\nu}\right) \leq\|x\|_{K^{m}}^{\nu}
$$

for every $x \in B_{m}(t)$ and $\nu \in \mathbb{N}$.

Observe that Theorem 1.1 is (by the local structure of $K$-analytic sets, Theorem 2.6) an immediate consequence of Theorem 4.1.

Proof of Theorem 4.1. We follow the idea of the proof of Theorem 4 from [3] where higher order approximation for classical analytic sets is considered.

Let $\psi: B_{m}(1) \rightarrow E$, where $E=\phi\left(\left(K^{k}\right)_{\text {sym }}^{d}\right)$, be a definable $K$-differentiable map (for the definitions of $\phi$ and $\left(K^{k}\right)_{\text {sym }}^{d}$ see Section 2.3. Next put $r=k d$ and identify the space $\left((K)^{k}\right)^{d}$ with $K^{r}$. Let $W\left(z_{1}, \ldots, z_{r}\right)=$ $\phi \circ \pi_{\mathrm{sym}}\left(z_{1}, \ldots, z_{r}\right)$ for $z_{i} \in K, i=1, \ldots, r$. $\left(\pi_{\mathrm{sym}}\right.$ is introduced in Section 2.3 here $\pi_{\text {sym }}$ is treated, via the identification above, as a mapping defined on $K^{r}$. Recall that $W$ is a polynomial mapping.) Now define

$$
X(\psi, t)=\left\{\left(x, z_{1}, \ldots, z_{r}\right) \in B_{m}(t) \times K^{r}: \psi(x)=W\left(z_{1}, \ldots, z_{r}\right)\right\} .
$$


Observe that the fact that the image of $\psi$ is contained in $E$, and the properties of $\phi$ imply that for $0<t \leq 1$ the set $X(\psi, t)$ is a purely $m$-dimensional $K$ analytic subset of $B_{m}(t) \times K^{r}$ with definably proper projection onto $B_{m}(t)$.

Let $Y$ be any purely $m$-dimensional $K$-analytic subset of $B_{m}(t) \times K^{k}$ with definably proper projection onto $B_{m}(t)$ such that the generic cardinality of the fiber of $Y$ over $B_{m}(t)$ equals $d$, and let pr: $K^{m} \times K^{k} \times K^{r-k} \rightarrow K^{m} \times K^{k}$ be the natural projection. Then there exists a $K$-differentiable mapping $\psi$ : $B_{m}(t) \rightarrow E$ such that $Y=\operatorname{pr}(X(\psi, t)$ ) (cf. [2] for the case $K=\mathbb{C}$ ). Indeed, let $S$ denote the set

$$
\left\{x \in B_{m}(t): \sharp\left(\left(\{x\} \times K^{k}\right) \cap Y\right)<d\right\},
$$

which is definable and has $\operatorname{dim}_{R}(S) \leq 2 m-2$ (see Theorem 2.6). Next let $a_{1}(x), \ldots, a_{d}(x)$, for every $x \in B_{m}(t) \backslash S$, be the points from $Y$ over $x$. By Theorem 2.6, every such $x$ has a neighborhood $U$ in $B_{m}(t)$ in which $a_{1}, \ldots, a_{d}$ are $K$-differentiable mappings. Let $\psi: B_{m}(t) \backslash S \rightarrow E$ be given by $\psi(x)=\left(\left\langle a_{1}(x), \ldots, a_{d}(x)\right\rangle\right)$. The Riemann theorem (see Theorem 2.1) implies that $\psi$ can be extended to a $K$-differentiable mapping defined on the whole $B_{m}(t)$. Clearly $Y=\operatorname{pr}(X(\psi, t))$.

The idea of the proof of Theorem 4.1 is to approximate the $K$-differentiable mapping $\psi$ such that $X=\operatorname{pr}(X(\psi, 1))$ by $K$-Nash mappings $\psi_{\nu}$ on $B_{m}(t)$. As we shall show, $\operatorname{pr}\left(X\left(\psi_{\nu}, t\right)\right)$ will be an approximation of $X \cap$ $\left(B_{m}(t) \times K^{k}\right)$ we look for. (Note that if $\psi_{\nu}$ are $K$-Nash mappings then, by Theorem 2.4 $\operatorname{pr}\left(X\left(\psi_{\nu}, t\right)\right)$ are $K$-Nash sets of pure $K$-dimension $m$.) More precisely, let $\psi: B_{m}(1) \rightarrow E$ be a $K$-differentiable mapping such that $X=\operatorname{pr}(X(\psi, 1))$. We shall prove that there are $t<1$ and a sequence $\psi_{\nu}: B_{m}(t) \rightarrow E$ of Nash mappings such that

$$
\rho\left(\left(\{x\} \times K^{k}\right) \cap \operatorname{pr}(X(\psi, t)),\left(\{x\} \times K^{k}\right) \cap \operatorname{pr}\left(X\left(\psi_{\nu}, t\right)\right)\right) \leq\|x\|_{K^{m}}^{\nu}
$$

for every $x \in B_{m}(t)$ and $\nu \in \mathbb{N}$.

To do this we reduce the problem to the case of analytic sets of codimension one. We shall need the following lemma and proposition (whose proofs are very similar to those in the classical situation (see [3]) and are recalled for completeness).

Lemma 4.2. Fix, $n, r \in \mathbb{N}$. There exist $K$-linear forms $L_{1}, \ldots, L_{s}: K^{r} \rightarrow K$ and a constant $C \in R, C>0$, such that for every $x_{1}, \ldots, x_{n}, y_{1}, \ldots, y_{n} \in K^{r}$ there is $i \in\{1, \ldots, s\}$ such that

$$
\begin{aligned}
\rho_{r}\left(\left\langle x_{1}, \ldots, x_{n}\right\rangle,\left\langle y_{1}, \ldots y_{n}\right\rangle\right) & \\
& \leq C \rho_{1}\left(\left\langle L_{i}\left(x_{1}\right), \ldots, L_{i}\left(x_{n}\right)\right\rangle,\left\langle L_{i}\left(y_{1}\right), \ldots, L_{i}\left(y_{n}\right)\right\rangle\right) .
\end{aligned}
$$

Proof of Lemma 4.2. The case $r=1$ is obvious, so assume $r>1$. We shall show the following condition: 
(a) $\exists L_{1}, \ldots, L_{s} \exists C>0 \forall X, Y \subset K^{r}, \sharp X, \sharp Y \leq n$

$$
\exists i \in\{1, \ldots, s\} \forall x \in X, y \in Y:\|x-y\| \leq C\left|L_{i}(x)-L_{i}(y)\right| .
$$

First let us check that once (a) is proved, the $L_{1}, \ldots, L_{s}, C$ obtained satisfy the assertion of the lemma. Suppose otherwise: there are $\left\langle x_{1}, \ldots, x_{n}\right\rangle$ and $\left\langle y_{1}, \ldots, y_{n}\right\rangle$ such that for every $i \in\{1, \ldots, s\}$,

$$
\begin{aligned}
\rho_{r}\left(\left\langle x_{1}, \ldots, x_{n}\right\rangle,\left\langle y_{1}, \ldots, y_{n}\right\rangle\right) & \\
& >C \rho_{1}\left(\left\langle L_{i}\left(x_{1}\right), \ldots, L_{i}\left(x_{n}\right)\right\rangle,\left\langle L_{i}\left(y_{1}\right), \ldots, L_{i}\left(y_{n}\right)\right\rangle\right) .
\end{aligned}
$$

This implies that for every $i \in\{1, \ldots, s\}$ and every permutation $p$ there is a permutation $q$ such that

$$
C \max _{j}\left|L_{i}\left(x_{j}\right)-L_{i}\left(y_{q(j)}\right)\right|<\max _{j}\left\|x_{j}-y_{p(j)}\right\| .
$$

For every $i$, let $q_{i}$ be a permutation such that $\max _{j}\left|L_{i}\left(x_{j}\right)-L_{i}\left(y_{q_{i}(j)}\right)\right|$ takes the smallest possible value. Then obviously, for every $i \in\{1, \ldots, s\}$,

$$
C \max _{j}\left|L_{i}\left(x_{j}\right)-L_{i}\left(y_{q_{i}(j)}\right)\right|<\max _{j}\left\|x_{j}-y_{q_{i}(j)}\right\| .
$$

Now, for every $i$, let $j_{i}$ be such that $\left\|x_{j_{i}}-y_{q_{i}\left(j_{i}\right)}\right\|$ takes the greatest possible value. Then for every $i \in\{1, \ldots, s\}$,

$$
C\left|L_{i}\left(x_{j_{i}}\right)-L_{i}\left(y_{q_{i}\left(j_{i}\right)}\right)\right|<\left\|x_{j_{i}}-y_{q_{i}\left(j_{i}\right)}\right\| \text {. }
$$

It follows that for $X=\left\{x_{1}, \ldots, x_{n}\right\}, Y=\left\{y_{1}, \ldots, y_{n}\right\}$ and for every $i \in$ $\{1, \ldots, s\}$ there is $(x, y) \in X \times Y$ such that

$$
C\left|L_{i}(x)-L_{i}(y)\right|<\|x-y\|,
$$

contrary to (a).

Now let us prove (a). It is easy to check that, since $r>1$, there exists a finite set $S$ of $(r-1)$-dimensional $K$-linear spaces $l_{1}, \ldots, l_{s} \subset K^{r}$ and there exists $\alpha \in R, \alpha>0$, with the following property:

(b) for all $X, Y \subset K^{r}$ with $\sharp X=\sharp Y=n$ there is $l \in S$ such that for all $(k, m) \in X \times Y$ with $m \neq k$,

$$
\operatorname{dist}\left(\frac{m-k}{\|m-k\|}, l\right)>\alpha \text {. }
$$

The set $S$ will be interpreted as the set of $K$-linear forms $L_{1}, \ldots, L_{s}$ which are defined as the projections of $K^{r}$ along $l_{1}, \ldots, l_{s}$ onto the orthogonal complements of $l_{1}, \ldots, l_{s}$, respectively. Then (b) easily implies that for such $L_{1}, \ldots, L_{s}$ there is $C=\alpha$ such that (a) is satisfied. Thus the proof of the lemma is complete.

For any $K$-linear form $L: K^{r} \rightarrow K$ define $\Phi_{L}: K^{m} \times K^{r} \rightarrow K^{m} \times K$ by $\Phi_{L}\left(x, z_{1}, \ldots, z_{r}\right)=\left(x, L\left(z_{1}, \ldots, z_{r}\right)\right)$. Note that for every definable $K$ differentiable mapping $\tilde{\psi}: B_{m}(t) \rightarrow E$, the set $\Phi_{L}(X(\tilde{\psi}, t))$ is a $K$-analytic 
subset of $B_{m}(t) \times K$ of codimension one with proper projection onto $B_{m}(t)$ (an immediate consequence of Theorem 2.4).

The following proposition will be useful.

Proposition 4.3. Let $\psi: B_{m}(1) \rightarrow E$ be a definable $K$-differentiable mapping. For every finite collection $L_{1}, \ldots, L_{s}: K^{r} \rightarrow K$ of $K$-linear forms there exist $t \in R, t>0$, and a sequence $\left\{\psi_{\nu}: B_{m}(t) \rightarrow E\right\}$ of $K-$ Nash mappings such that for every $x \in B_{m}(t), i \in\{1, \ldots, s\}$ and every $\nu \in \mathbb{N}$,

$$
\rho\left((\{x\} \times K) \cap \Phi_{L_{i}}\left(X\left(\psi_{\nu}, t\right)\right),(\{x\} \times K) \cap \Phi_{L_{i}}(X(\psi, t))\right) \leq\|x\|_{K^{m}}^{\nu} .
$$

Proof of Proposition 4.3. We shall need the following two lemmas.

Lemma 4.4. Let $p(x, z)=z^{k}+a_{1}(x) z^{k-1}+\cdots+a_{k}(x)$ and $q(x, z)=$ $z^{k}+b_{1}(x) z^{k-1}+\cdots+b_{k}(x)$ be polynomials with definable coefficients which are $K$-differentiable on $B_{m}(s), s>0$. Assume $a_{i}(0)=0$ for $i=1, \ldots, k$ and there exists $\nu \in \mathbb{N}$ such that $\left|a_{i}(x)-b_{i}(x)\right| \leq\|x\|_{K^{m}}^{\nu}$ for all $i \in\{1, \ldots, k\}$ and $x \in B_{m}(s)$. Let $w_{1}(x), \ldots, w_{k}(x)$ and $v_{1}(x), \ldots, v_{k}(x)$ denote the roots of $p(x, z)$ and $q(x, z)$ respectively. Then there is $0<t \leq s$ such that for every integer $\mu$ there is $\nu_{0}$ such that if $\nu>\nu_{0}$ then

$$
\rho\left(\left\langle w_{1}(x), \ldots, w_{k}(x)\right\rangle,\left\langle v_{1}(x), \ldots, v_{k}(x)\right\rangle\right) \leq\|x\|_{K^{m}}^{\mu}
$$

for every $x \in B_{m}(t)$.

Proof of Lemma 4.4. Let $0<t \leq s$ be such that $\left|w_{i}(x)\right|<1$ for all $i \in\{1, \ldots, k\}$ and $x \in B_{m}(t)$. Fix $\mu \in \mathbb{N}$ and for every $x \in B_{m}(t)$ put $E_{x}=\bigcup_{j=1}^{k} K\left(w_{j}(x),\|x\|^{\mu} / 4 k\right)$, where $K(a, r)=\{z \in K:|z-a|<r\}$. Then $E_{x}=E_{x}^{1} \cup \cdots \cup E_{x}^{s(x)}$, where $E_{x}^{i}$ is a definably connected component of $E_{x}$ for $i \in\{1, \ldots, s(x)\}$. Moreover, for $i \in\{1, \ldots, s(x)\}$ and $j \in\{1, \ldots, k\}$,

$(\mathrm{x}) \operatorname{dist}\left(w_{j}(x), \partial E_{x}^{i}\right) \geq\|x\|^{\mu} / 4 k$,

(xx) $\operatorname{diam} E_{x}^{i} \leq\|x\|^{\mu} / 2$.

By (x) we have $\inf _{\partial E_{x}^{i}}|p(x, \cdot)| \geq\left(\|x\|^{\mu} / 4 k\right)^{k}$. Now, by the choice of $t$, we can take $\nu_{0}$ so large that if $\nu>\nu_{0}$ then $\sup _{\partial E_{x}^{i}}|p(x, \cdot)-q(x, \cdot)|<\left(\|x\|^{\mu} / 4 k\right)^{k}$. Then, by a definable version of the Rouché theorem (see [11, p. 435]), for every $x \in B_{m}(t)$ and $i \in\{1, \ldots, s(x)\}$, the numbers of the roots of $p(x, z)$ and $q(x, z)$ (counted with multiplicities) in $E_{x}^{i}$ are equal. In view of (xx) we are done.

Lemma 4.5. For every $K$-linear form $L: K^{r} \rightarrow K$ there are polynomials $P_{L, 1}, \ldots, P_{L, n_{L}} \in K\left[y_{1}, \ldots, y_{N}, z\right]$ such that for any definable $K$ differentiable mapping $\psi: B_{m}(1) \rightarrow E \subset K^{N}$,

$$
\begin{aligned}
& \Phi_{L}(X(\psi, t)) \\
& \quad=\left\{(x, z) \in B_{m}(t) \times K: P_{L, 1}(\psi(x), z)=\cdots=P_{L, n_{L}}(\psi(x), z)=0\right\}
\end{aligned}
$$

for $t<1$. 
Proof of Lemma 4.5. The mapping $\psi$ is of the form $\psi=\left(\psi_{1}, \ldots, \psi_{N}\right)$. Put $y=\left(y_{1}, \ldots, y_{N}\right)$ and define

$$
V=\left\{\left(y, z_{1}, \ldots, z_{r}\right) \in K^{N} \times K^{r}: y=W\left(z_{1}, \ldots, z_{r}\right)\right\} .
$$

Then $\Phi_{L}(V)$ is an algebraic subset of $K^{N} \times K$ (because it is the image of an algebraic subset of $K^{N} \times K^{r}$ under a definably proper polynomial mapping). Let $P_{L, 1}, \ldots, P_{L, n_{L}} \in K\left[y_{1}, \ldots, y_{N}, z\right]$ be polynomials describing $\Phi_{L}(V)$. It is easy to see that these polynomials satisfy the assertion of the lemma.

Proof of Proposition 4.3 (continuation). Fix $K$-linear forms $L_{1}, \ldots, L_{s}$. For these forms, we shall construct a system of polynomial equations and a system of definable $K$-differentiable (in our o-minimal structure) functions satisfying the equations. (Among the functions there will be the components of $\psi$.) Next the solutions to the system will be approximated by $K$-Nash functions (by means of Theorem 3.1). It will turn out that the resulting approximations of $\psi$ satisfy the assertion of the proposition.

First, there exists $Q=\left(Q_{1}, \ldots, Q_{\tilde{d}}\right), Q_{j} \in K\left[y_{1}, \ldots, y_{N}\right]$ for $j \in\{1, \ldots, \tilde{d}\}$, such that $E=\{Q=0\}$. Then $\psi: B_{m}(1) \rightarrow E \subset K^{N}$ satisfies $Q(\psi(x))=0$ for $x \in B_{m}(1)$.

Next for every $L \in\left\{L_{1}, \ldots, L_{s}\right\}$, there are $P_{L, i} \in K\left[y_{1}, \ldots, y_{N}, z\right]$ as in Lemma 4.5. Then by the Weierstrass preparation theorem we can write

$$
P_{L, i}(\psi(x), z)=H_{L, i}(x, z)\left(W_{L}(x, z)\right)^{k_{L, i}} \quad \text { for } i=1, \ldots, n_{L} .
$$

Here $W_{L}$ is the optimal polynomial for $\Phi_{L}(X(\psi, 1))$ in $z$ (i.e. the unitary polynomial in $z$ with definable $K$-differentiable coefficients and non-zero discriminant, describing $\left.\Phi_{L}(X(\psi, 1))\right), H_{L, i}$ is a polynomial in $z$ such that $\left\{W_{L}=0\right\}$ is not contained in $\left\{H_{L, i}=0\right\}$, and $k_{L, i}$ is an integer. (The existence of $W_{L}(x, z)$ follows from the fact that $\Phi_{L}(X(\psi, 1))$ is of codimension one with definably proper projection onto $B_{m}(1)$, which is a consequence of Theorem 2.4. Indeed, let $p_{1}(x), \ldots, p_{d}(x) \in K$ be such that $\left\{\left(x, p_{j}(x)\right): j=1, \ldots, d\right\}=\Phi_{L}(X(\psi, 1)) \cap\left(\{x\} \times K^{k}\right)$ and define $W_{L}(x, z)=$ $\left(z-p_{1}(x)\right) \cdots\left(z-p_{d}(x)\right)$. By Theorem 2.6 the coefficients of $W_{L}(x, z)=$ $z^{d}+z^{d-1} c_{L, 1}(x)+\cdots+c_{L, d}(x)$ are $K$-differentiable functions in a neighborhood of every $x \in B_{m}(1)$ such that $p_{j}(x) \neq p_{i}(x)$ for $i \neq j$. Then by Theorem 2.1 the coefficients are $K$-differentiable on $B_{m}(1)$.)

Consider the system of polynomial equations

$$
\begin{aligned}
& Q(\psi(x))=0, \\
& P_{L_{j}, i}(\psi(x), z)=H_{L_{j}, i}(x, z)\left(z^{d}+z^{d-1} c_{L_{j}, 1}(x)+\cdots+c_{L_{j}, d}(x)\right)^{k_{L_{j}, i}}
\end{aligned}
$$

for $j \in\{1, \ldots, s\}$ and $i \in\left\{1, \ldots, n_{L_{j}}\right\}$, satisfied by the (definable $K$ differentiable) mappings $\psi$ and coefficients of $W_{L_{j}}, H_{L_{j}, i}$.

By Theorem 3.1 there are $0<t<1$ and $K$-Nash mappings $\psi_{\nu}, c_{L_{j}, 1, \nu}$, $\ldots, c_{L_{j}, d, \nu}$ sufficiently close to $\psi, c_{L_{j}, 1}, \ldots, c_{L_{j}, d}$ on $B_{m}(t)$, satisfying the 
system $(\mathrm{P})$. (Formally, we should get rid of the variable $z$ before we use the approximation theorem. Yet, since $P_{L_{j}, i}, H_{L_{j}, i}, W_{L_{j}}$ are polynomials in $z$, each equation $P_{L_{j}, i}=H_{L_{j}, i} \cdot\left(W_{L_{j}}\right)^{k_{L_{j}, i}}$ is equivalent to a finite number of polynomial equations in which $z$ does not appear, obtained by comparing the coefficients of $P_{L_{j}, i}$ and $H_{L_{j}, i} \cdot\left(W_{L_{j}}\right)^{k_{L_{j}, i}}$.)

One easily observes that the polynomials $W_{L, \nu}$ defined by replacing $c_{L, 1}, \ldots, c_{L, d}$ in $W_{L}$ by $c_{L, 1, \nu}, \ldots, c_{L, d, \nu}$ are the optimal polynomials for $\Phi_{L}\left(X\left(\psi_{\nu}, t\right)\right)$, where $L \in\left\{L_{1}, \ldots, L_{s}\right\}$. Choosing the $K$-Nash solutions to the system $(\mathrm{P})$ sufficiently close to the original $K$-differentiable solutions, by Lemma 4.4 applied to $W_{L, \nu}$ and $W_{L}$, we see that the mappings $\psi_{\nu}$ satisfy the assertion of the proposition.

Proof of Theorem 4.1 (end). Without loss of generality we may assume $X \cap\left(\{0\}^{m} \times K^{k}\right)=\{0\}^{m+k}$. Recall that $\psi: B_{m}(1) \rightarrow E$ is a $K$-differentiable mapping such that $\operatorname{pr}(X(\psi, 1))=X$ whereas $d$ is the generic cardinality of the fiber of $X$ over $B_{m}(1)$. Next apply Lemma 4.2 with $n=d$ and $r=k$ to obtain $K$-linear forms $L_{1}, \ldots, L_{s}: K^{k} \rightarrow K$ satisfying the assertion of that lemma.

Note that for every $i \in\{1, \ldots, s\}$ there is a $K$-linear form $\tilde{L}_{i}:\left(K^{k}\right)^{d} \rightarrow$ $K$ such that $\Phi_{\tilde{L}_{i}}=\Phi_{L_{i}} \circ$ pr. Using Proposition 4.3 we obtain a sequence of $K$-Nash mappings $\psi_{\nu}: B_{m}(t) \rightarrow E$ such that for all $x \in B_{m}(t), i \in\{1, \ldots, s\}$ and $\nu \in \mathbb{N}$,

$$
\rho\left((\{x\} \times K) \cap \Phi_{\tilde{L}_{i}}\left(X\left(\psi_{\nu}, t\right)\right),(\{x\} \times K) \cap \Phi_{\tilde{L}_{i}}(X(\psi, t))\right) \leq\|x\|_{K^{m}}^{\nu} .
$$

First let us check that $X_{\nu}=\operatorname{pr}\left(X\left(\psi_{\nu}, t\right)\right)$ has $d$ elements in the generic fiber over $B_{m}(t)$ for almost all $\nu$. Since $\psi_{\nu}\left(B_{m}(t)\right) \subset E$, every fiber may have at most $d$ elements. This number equals $d$ precisely because $\Phi_{L_{i}}\left(\operatorname{pr}\left(X\left(\psi_{\nu}, t\right)\right)\right)$ have $d$ elements in the generic fibers over $B_{m}(t)$ for almost all $\nu$ as they are described by polynomials of degree $d$ with non-zero discriminants.

Let us prove that the required bound holds. By Lemma 4.2, for every $x \in B_{m}(t)$ and $\nu \in \mathbb{N}$ there is $i \in\{1, \ldots, s\}$ such that

$$
\begin{aligned}
& \rho\left(\left(\{x\} \times K^{k}\right) \cap \operatorname{pr}(X(\psi, t)),\left(\{x\} \times K^{k}\right) \cap \operatorname{pr}\left(X\left(\psi_{\nu}, t\right)\right)\right) \\
& \quad \leq C \cdot \rho\left((\{x\} \times K) \cap \Phi_{\tilde{L}_{i}}\left(X\left(\psi_{\nu}, t\right)\right),(\{x\} \times K) \cap \Phi_{\tilde{L_{i}}}(X(\psi, t))\right),
\end{aligned}
$$

where $C$ is a positive constant. Now, replacing $\psi_{\nu}$ by $\psi_{\nu+1}$ and decreasing $t$ if necessary so that $\|x\|^{\nu+1} \leq C\|x\|^{\nu}$ for every $x \in B_{m}(t)$, we get the required inequality.

Acknowledgments. The first named author is indebted to Professor Sergei Starchenko for stimulating discussions during the programme on Model Theory in Cambridge in 2005. He also thanks the Newton Mathematical Institute for hospitality. 


\section{References}

[1] M. André, Cinq exposés sur la désingularisation, manuscript, École Polytechnique Fédérale de Lausanne, 1992.

[2] M. Bilski, Approximation of analytic sets with proper projection by Nash sets, C. R. Math. Acad. Sci. Paris 341 (2005), 747-750.

[3] - Approximation of analytic sets by Nash tangents of higher order, Math. Z. 256 (2007), 705-716.

[4] - Algebraic approximation of analytic sets and mappings, J. Math. Pures Appl. 90 (2008), 312-327.

[5] E. Chirka, Complex Analytic Sets, Kluwer, Dordrecht, 1989.

[6] M. Coste, J. Ruiz and M. Shiota, Approximation in compact Nash manifolds, Amer. J. Math. 117 (1995), 905-927.

[7] L. van den Dries, Tame Topology and o-Minimal Structures, London Math. Soc. Lecture Note Ser. 248, Cambridge Univ. Press, Cambridge, 1998.

[8] L. Lempert, Algebraic approximations in analytic geometry, Invent. Math. 121 (1995), 335-354.

[9] H. Matsumura, Commutative Algebra, Benjamin, New York, 1970.

[10] T. Ogoma, General Néron desingularization based on the idea of Popescu, J. Algebra 167 (1994), 57-84.

[11] Y. Peterzil and S. Starchenko, Expansions of algebraically closed fields in o-minimal structures, Selecta Math. 7 (2001), 409-445.

[12] - - - Expansions of algebraically closed fields II: Functions of several variables, J. Math. Logic 3 (2003), 1-35.

[13] - , 一, Complex analytic geometry in a non-standard setting, in: Model Theory with Applications to Algebra and Analysis, Vol. I, Cambridge Univ. Press, Cambridge, 2008, 117-165.

[14] D. Popescu, General Néron desingularization, Nagoya Math. J. 100 (1985), 97-126.

[15] —, General Néron desingularization and approximation, ibid. 104 (1986), 85-115.

[16] M. Spivakovsky, A new proof of D. Popescu's theorem on smoothing of ring homomorphisms, J. Amer. Math. Soc. 12 (1999), 381-444.

[17] P. Tworzewski, Intersections of analytic sets with linear subspaces, Ann. Scuola Norm. Sup. Pisa 17 (1990), 227-271.

[18] H. Whitney, Complex Analytic Varieties, Addison-Wesley, Reading, MA, 1972.

[19] G. T. Whyburn, Topological Analysis, Princeton Univ. Press, Princeton, 1964.

Marcin Bilski, Kamil Rusek

Institute of Mathematics

Jagiellonian University

Łojasiewicza 6

30-348 Kraków, Poland

E-mail: Marcin.Bilski@im.uj.edu.pl

Kamil.Rusek@im.uj.edu.pl

Received 12.8.2008

and in final form 17.5.2009 